\title{
Changes in lymphocyte subsets and functions in spleen from mice with high fat diet-induced obesity
}

\author{
G. C. Baccan ${ }^{1,2}$, O. Hernández ${ }^{3}$, L. E. Díaz ${ }^{1}$, A. Gheorghe ${ }^{1}$, T. Pozo-Rubio ${ }^{1}$, A. Marcos ${ }^{1}$ and \\ M. De La Fuente \\ ${ }^{1}$ Department of Metabolism and Nutrition. Institute of Food Science, Technology and Nutrition (ICTAN), Spanish National \\ Research Council (CSIC), Madrid, Spain, ${ }^{2}$ Department of Biofunction, Institute of Health Sciences, Federal University of \\ Bahia (UFBA), Salvador, Brazi and ${ }^{3}$ Department of Physiology. Faculty of Biology, Complutense University of Madrid
}

Obesity is associated with a low-grade inflammation state that contributes to an increased risk of developing cardiovascular disease and other pathologies, in which the immune system alterations are involved. The aim of this study was to find out the effects of obesity in adult mice through the intake of a high fat diet during their adolescence on several spleen characteristics such as size, number of lymphocyte subsets and immune cell functions. Young females ICR mice $(n=16)$ (14 weeks old) were divided into two groups $(n=8)$. The experimental group was fed ad libitum with a high fat diet (HFD) (60\% fat) (Harlan). The control group (C) was fed a maintenance diet. Food and water intake as well as body weights were determined twice per week. After 14 weeks the mice were sacrificed and the spleens were collected and weighed. Then, leukocytes from spleen were obtained and the different lymphocyte subsets analysed by flow cytometry. The following functions were also evaluated: the chemotaxis capacity, NK cell activity and the proliferative response of lymphocytes to mitogens such as Con A and LPS. HFD ingestion caused increased spleen size $(0.190 \pm 0.074 v s 0.122 \pm 0.016$, (g) $P=0.026)$ and $\mathrm{CD} 45+\mathrm{CD} 3+/ \mathrm{CD} 19+$ ratio values $(0.54 \pm 0.30$ vs $0.18 \pm 0.14, P=0.048)$ in comparison with the control group. Obese mice showed a decrease in NK cell activity $(33 \pm 12$ vs $60 \pm 11$ (\% lysis), $P<0.001)$ and an increase in spleen lymphocyte chemotaxis $(953 \pm 134$ vs $532 \pm 68, P<0.001)$. The treatment with a $60 \%$ fat-rich diet during adolescence produces changes in the mouse spleen in adulthood, which could be related to their obesity and may be a cause of the development of chronic pathologies.

Support: MICINN (BFU2011-30336), UCM (910379ENEROINN) grants and RETICEF (RD06/0013/0003) from ISCIII and BTSA (PRONAOS Study, CDTI 2008 1114). 